\title{
Synthesis and Cytotoxicity of Novel Sansalvamide A Derivatives
}

Chris L. Carroll, Jennifer V. C. Johnston, Ahmet Kekec, Joseph D. Brown, Emily Parry, Julia Cajia, Irene Medina, Kristina M. Cook, Po-Shen Pan, and Shelli R. McAlpine*

Department of Chemistry and Biochemistry, 5500 Campanile Drive, San Diego State University, San Diego, CA 92182.

\section{Synthesis}

\section{SUPPORTING INFORMATION}

General Remarks. All coupling reactions were performed under argon atmosphere with the exclusion of moisture. All reagents were used as received. Anhydrous methylene chloride Dri Solv (EM) and Anhydrous Acetonitrile Dri Solv (EM) were bought from VWR, and were packed under nitrogen with a septum cap. Diisopropylethylamine (DIPEA) was purchased from Aldrich, packaged under nitrogen in a sure seal bottle. The coupling agent HATU and PyAOP were purchased from Perspective:Applied Biosystems at 850 Lincoln Center Dr. Foster City, CA 94404, Telephone: 1-800-327-3002. The coupling agents TBTU, and PyBROP were purchased from NovaBiochem. DEPBT [3(diethoxyphosphoryloxy)-1,2,3-bezotriazine$4(3 \mathrm{H})$ ] was purchased from Aldrich (order number 49596-4). The ${ }^{1} \mathrm{H}$ NMR spectra were recorded on a Varian at $500 \mathrm{MHz}$. LCMS were obtained at San Diego State University using HP1100 Finnnigan LCQ. Flash column chromatography was performed on 230-400 mesh 32-74 $\mu \mathrm{m} 60$ Å silica gel from Bodman Industries.

General peptide synthesis. All peptide coupling reactions were carried out under argon with dried solvent, using methylene chloride for dipeptide and tripeptide couplings and acetonitrile for all other peptide couplings. The amine (1.1 equivalents) and acid ( 1 equivalent) were weighed into a dry flask along with 3 equivalents of DIPEA and 1.1 equivalents of TBTU.* Anhydrous methylene chloride was added for a $0.1 \mathrm{M}$ solution. The solution was stirred at room temperature and reactions were monitored by TLC. Reactions were run for 4 - 24 hours before working up by washing with saturated ammonium chloride. (Note: if acetonitrile was used for the reaction, methylene chloride was added to reaction upon workup and then the resulting solution was washed with ammonium chloride). After back extraction of aqueous layers with methylene chloride, organic layers were combined, dried over sodium sulfate, filtered and concentrated. Flash chromatography using $0-100 \%$ ethyl actetate-hexane gave our desired peptide.

* Some coupling reactions would not go to completion using only TBTU and therefore HATU, and/or DEPBT were used. In a few cases 1.1 equivalents of all three coupling reagents were used.

General Amine deprotection. Amines were deprotected using 20\% TFA in methylene chloride $(0.1 \mathrm{M})$ with two equivalents of anisole. The reactions were monitored by TLC, where the TLC sample was first worked up in a mini-workup using DI water and methylene chloride to remove TFA. Reactions were allowed to run for 1-2 hours and then concentrated in vacuo.

General Acid deprotection. Acids were deprotected using 4 equivalents of lithium hydroxide (or enough was added until $\mathrm{pH}=\sim 11)$ in methanol $(0.1 \mathrm{M})$. The peptide was placed in a flask, along with lithium hydroxide and methanol and stirred overnight. Within 12 hours the acid was usually deprotected. Work-up of reactions involved the acidification of reaction solution using $\mathrm{HCl}$ to $\mathrm{pH}=1$. The aqueous solution was extracted three times with methylene chloride, and the combined organic layer was dried, filtered and concentrated in vacuo.

Macrocyclization procedure (in situ). All pentapeptides were deprotected using $\mathrm{HCl}$ in methanol and the presence of the free amine was verified using LCMS. The reaction was then neutralized with Lithium hydroxide. Upon neutralization, $\mathrm{LiOH}$ was added ( $\sim$ equiv) to bring $\mathrm{pH}$ up to $\sim 11$. The acid deprotection was verified via LCMS. Upon acid deprotection the reaction was concentrated in vacuo and the crude, dry, 
double deprotected peptide (free acid and free amine) was dissolved in a minimum of dry acetonitrile.

Three coupling agents were initially used: DEPBT, HATU, and PyBROP ( 0.5 to 0.75 equivalents each). These coupling agents were dissolved in a calculated volume of dry $50 \%$ acetonitrile and $50 \%$ methylene chloride that would give a $0.01 \mathrm{M}$ solution when including the volume used for the deprotected peptide. The coupling agents were then added to the deprotected peptide solution. Three-five equivalents of DIPEA were then added to the reaction to ensure the $\mathrm{pH}$ was kept at or greater than 8 . If the solution was not clear, DMF or methylene chloride were added but no more than $20 \%$ of the volume used for the overall reaction. Note: in some cases methylene chloride addition improved the solution clarity more than DMF, this depended on the number of methyl groups on the compound (ie the hydrophobicity). With at least one methyl group it was found methylene chloride was a better solvent than DMF for clarity, with no methyl groups, DMF was found to be a better solvent for solution clarity. It is important to recognize that the coupling agents are typically not very soluble in acetonitrile, which is why an additional solvent is often used.

After 24 hours, TLC and LCMS (where the LCMS sample was worked up prior to injection) were taken, if no clear distinct product spot was visible, then typically PyAOP was added ( 0.5 equivalents), and sometimes, depending on reaction clarity 0.5 equivalents of HATU were also added. The comparison for Rf value in the product spot on TLC was the protected linear pentapeptide. The reaction was allowed to run another 24 hours, and checked again by TLC and LCMS. If the reaction was still not showing a clear product spot, then usually 0.25 equivalents of DEPBT were added and the reaction was allowed to run for 24-48 hours. At this point we found the reaction always demonstrated a product spot, although it was sometimes difficult to determine if it was complete (monitoring the starting material deprotected pentapeptide via LCMS was the easiest method). Upon completion, the reaction was worked up by washing with ammonium chloride. After back extraction of aqueous layers with methylene chloride, organic layers were combined, dried over sodium sulfate, filtered and concentrated. All macrocycles were purified using reverse phase HPLC, and a gradient of acetonitrile and DI water with $0.1 \%$ TFA.

\section{Bioassays: Protocol for HCT-116 Cytotoxcity Assay}

\section{Materials:}

1. Media

1000 mL McCoy's 5A Medium

$114 \mathrm{~mL}$ Fetal Bovine Serum

$25 \mathrm{~mL}$ Hepes buffer solution (1M)

$12.5 \mathrm{~mL}$ Penicillin- Streptomycin solution

2. Cell line

HCT-116, human colon cancer cell line.

3. Indicator

MTS and PMS in DPBS

4. $0.25 \%$ Trypsin- EDTA

5. Standard Anticancer drug

Etoposide (VP-16)

Stock is $4 \mathrm{mg} / \mathrm{mL}$ in DMSO

$\mathrm{IC}_{50} \sim 0.29-2.9 \mu \mathrm{g} / \mathrm{mL}$

6. DPBS (w/out $\mathrm{CaCl} \& \mathrm{MgCl}$ )

\section{Method:}

\section{Plating Cells}

1. Remove medium from the cells and rinse with $10 \mathrm{~mL}$ sterile DPBS.

2. Add $5 \mathrm{~mL}$ trypsin. 
3. Allow to stand in hood for 1 minute.

4. Remove trypsin with sterile pipette.

5. Allow flask to stand in hood for 2-5 minutes.

6. Add $10 \mathrm{~mL}$ of media to flask and pipette up and down to gently break up cell clumps.

7. Count the number of cells using a hemacytometer.

8. Determine \# of plates needed, and total volume of cell suspension ( 16 or $18 \mathrm{~mL}$ per plate).

9. Dilute cells to a final concentration of $2.5 \times 10^{4}$ cells $/ \mathrm{mL}$ in a sterile flask.

10. Pour cell suspension into a sterile trough.

For Serial Dilution Plates

1. Add $175 \mu \mathrm{L}$ to each well in the first row of a 96 well plate.

2. Add $150 \mu \mathrm{L}$ to the rest of the wells in A1-A10 \& A12.

3. In row 11 add $150 \mu \mathrm{L}$ cell suspension to the first 6 wells and only media to the last two.

For Single Concentration Plates

1. Add $150 \mu \mathrm{L}$ of sterile media to G11 \& H11.

2. Add $150 \mu \mathrm{L}$ of cell suspension to all other wells.

3. Add $25 \mu \mathrm{L}$ more to well $\mathrm{H} 1$.

Incubate plates overnight at $37^{\circ} \mathrm{C} \& 5 \% \mathrm{CO}_{2}$

\section{Assay}

For Pure Compounds \& Fractions

1. Add $25 \mu \mathrm{L}$ of test compounds @ $10 \mathrm{mg} / \mathrm{mL}$ to A1-A10, $25 \mu \mathrm{L}$ of DMSO to A11 \& $25 \mu \mathrm{L}$ of etoposide $(4 \mathrm{mg} / \mathrm{mL})$ to A12.

2. Using a twelve channel pipette, mix well then remove $50 \mu \mathrm{L}$ from the first row and transfer to the next row, repeat down the plate until row $\mathrm{F}$, then remove tip \# 11 and continue down plate discarding the last $50 \mu \mathrm{L}$.

For Crude Extracts

1. Make a dilution plate for each SIO box to be tested, add $195 \mu \mathrm{L}$ of media to 1-11 in all rows.

2. Add $5 \mu \mathrm{L}$ of crude extracts from box to dilution plate, and $5 \mu \mathrm{L}$ DMSO to column 11 .

3. Mix well, then add $6 \mu \mathrm{L}$ from dilution plate to test plate.

4. Add $25 \mu \mathrm{L}$ etoposide to $\mathrm{A} 12$. Remove $50 \mu \mathrm{L}$ and transfer down the plate.

Incubate plates for 72 hours.

Work up Using MTS

1. Add $20 \mu \mathrm{L}$ of MTS/PMS solution to each well.

2. Incubate for 3 hours.

3. Read plates@490 nm on plate reader.

HCT-116 Assay. The HCT-116 cells were maintained in McCoy's 5A media (Gibco) with $57.5 \mathrm{ml}$ fetal bovine serum (Bio Whittaker), $12.5 \mathrm{ml}$ IM HEPES (Fisher), $6.25 \mathrm{ml}$ penicillin-streptomycin (Gibco) at $37^{\circ} \mathrm{C}$ in $5 \% \mathrm{CO} 2 /$ air in tissue culture flasks. The cells were added to microtiter plates 24 hours prior to testing. Test materials were added to the plate and etoposide at $4 \mathrm{mg} / \mathrm{ml}$ (Sigma) and the solvent DMSO were used as positive and negative controls, respectively. These materials were then serially diluted (4- 
fold), and incubated for 72 hours. The cell viability was determined through the use of a colorimetric CellTiter 96 AQueous nonradioactive cell proliferation assay (Promega) where MTS (3-(4,5-

dimethylthiazol-2-yl)-5-(3-carboxymethoxyphenyl)-2-(4-sulfophenyl)-2H-tetrazolium, inner salt) and an electron coupling reagent PMS (phenazine mehosulfate) were bioreduced to formazan. This product was measured directly at 490nm using a Molecular Devices Emax microplate reader, and the inhibition concentration $\left(\mathrm{IC}_{50}\right)$ values were calculated by the analysis program SOFTmax PRO. The $\mathrm{IC}_{50}$ of etoposide is $0.29-2.9 \mu \mathrm{g} / \mathrm{ml}$. 


\section{LCMS Data for Final Cyclized Sansalvamide A Derivatives}
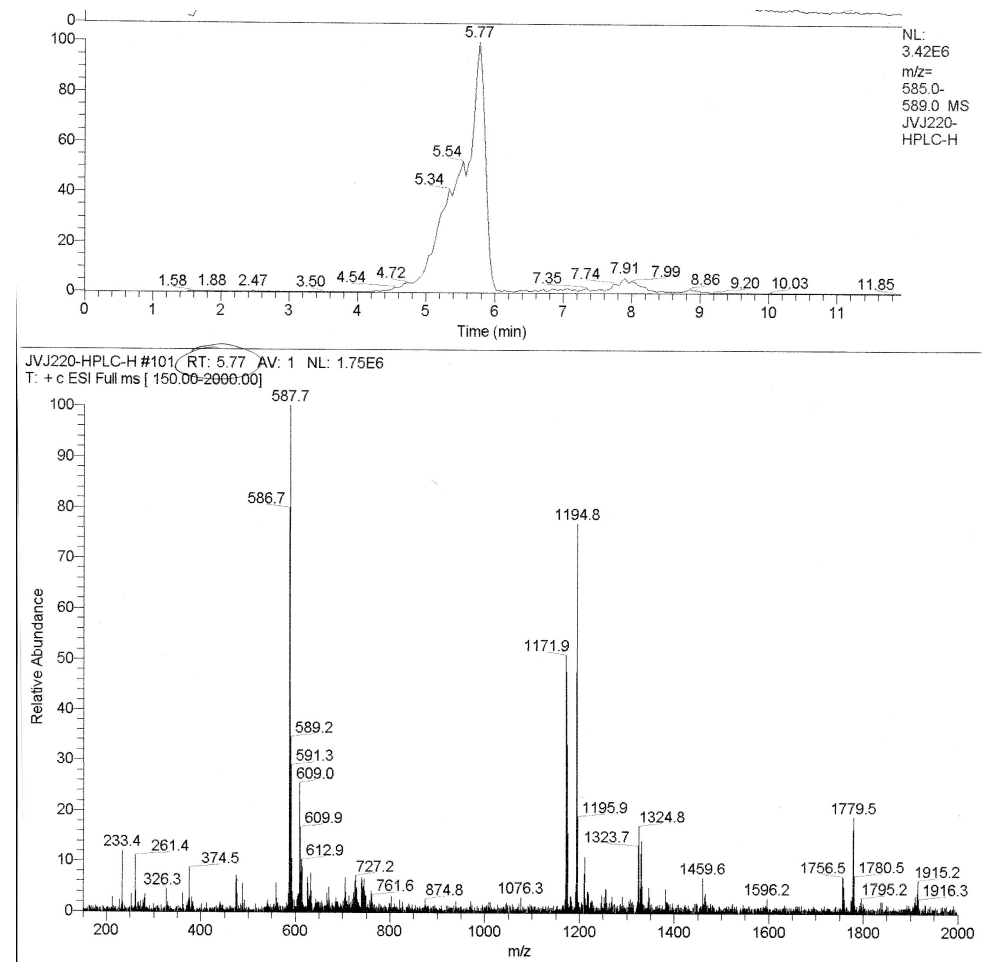

Compound A Cyclized 1a-2a-3a-4a-5a (MW=585)
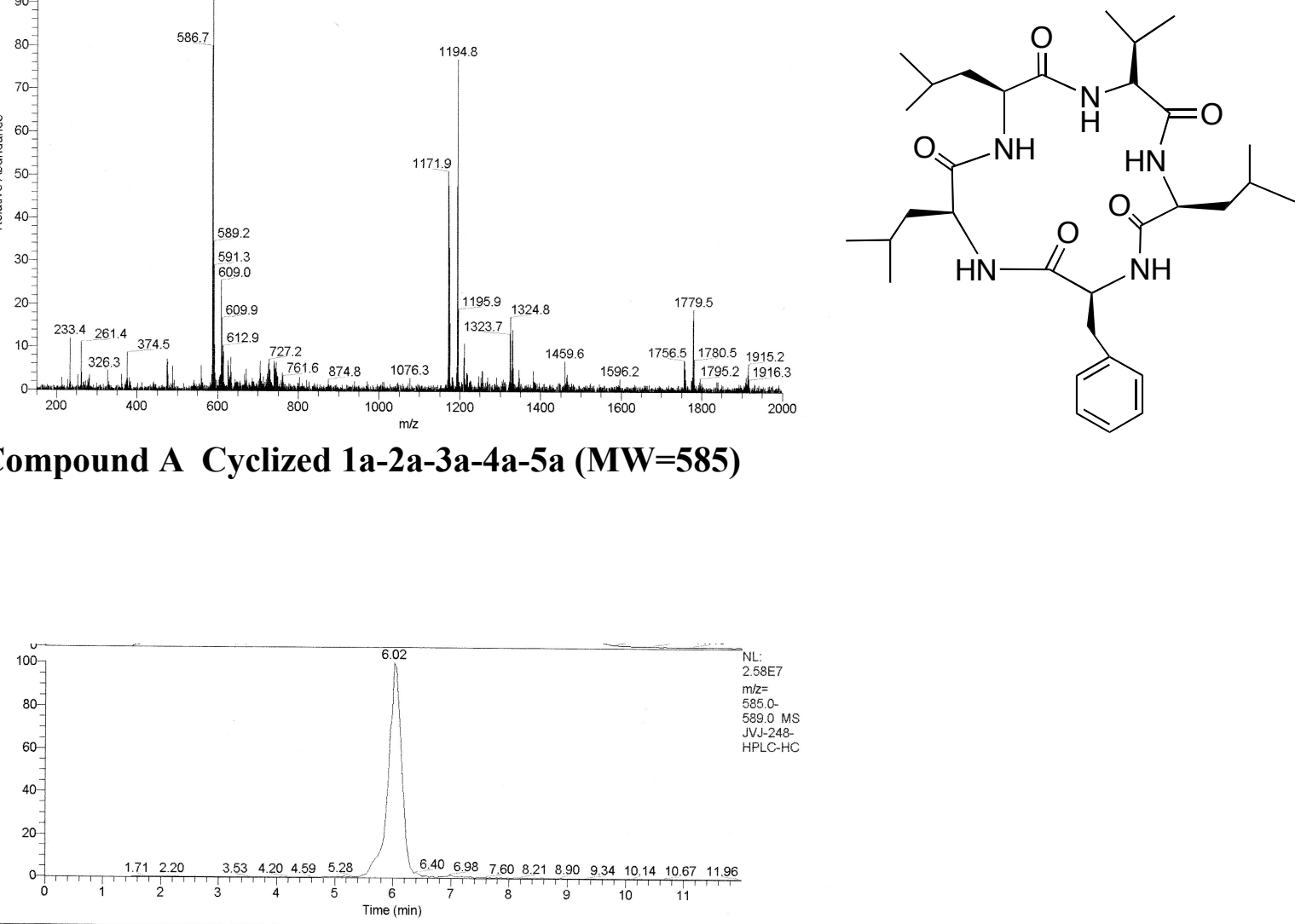

JVJ-248-HPLC-HC \#114 RT: 6.09 AV: 1 NL: $1.10 E 7$

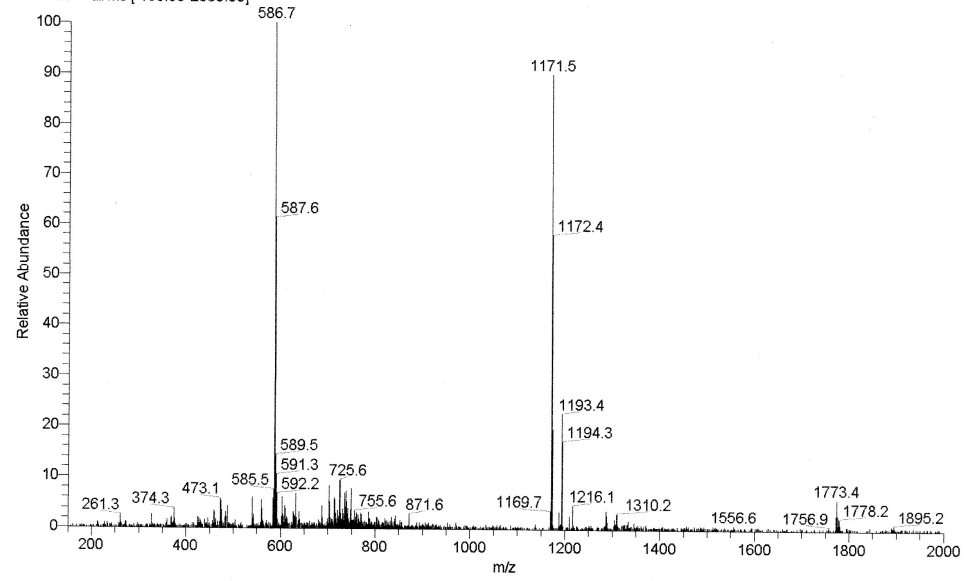

Compound B Cyclized 1b-2b-3b-4b-5b (MW=585)

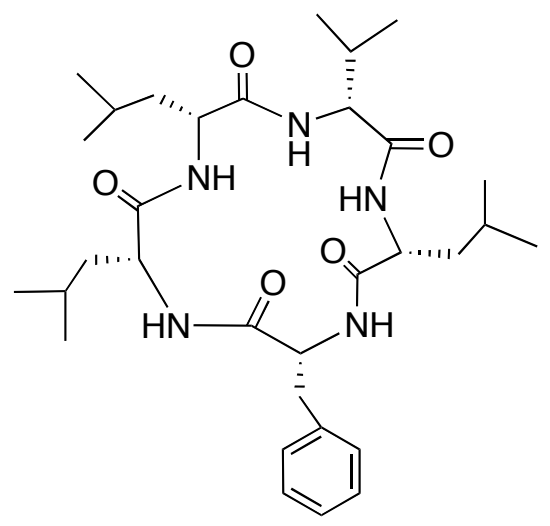



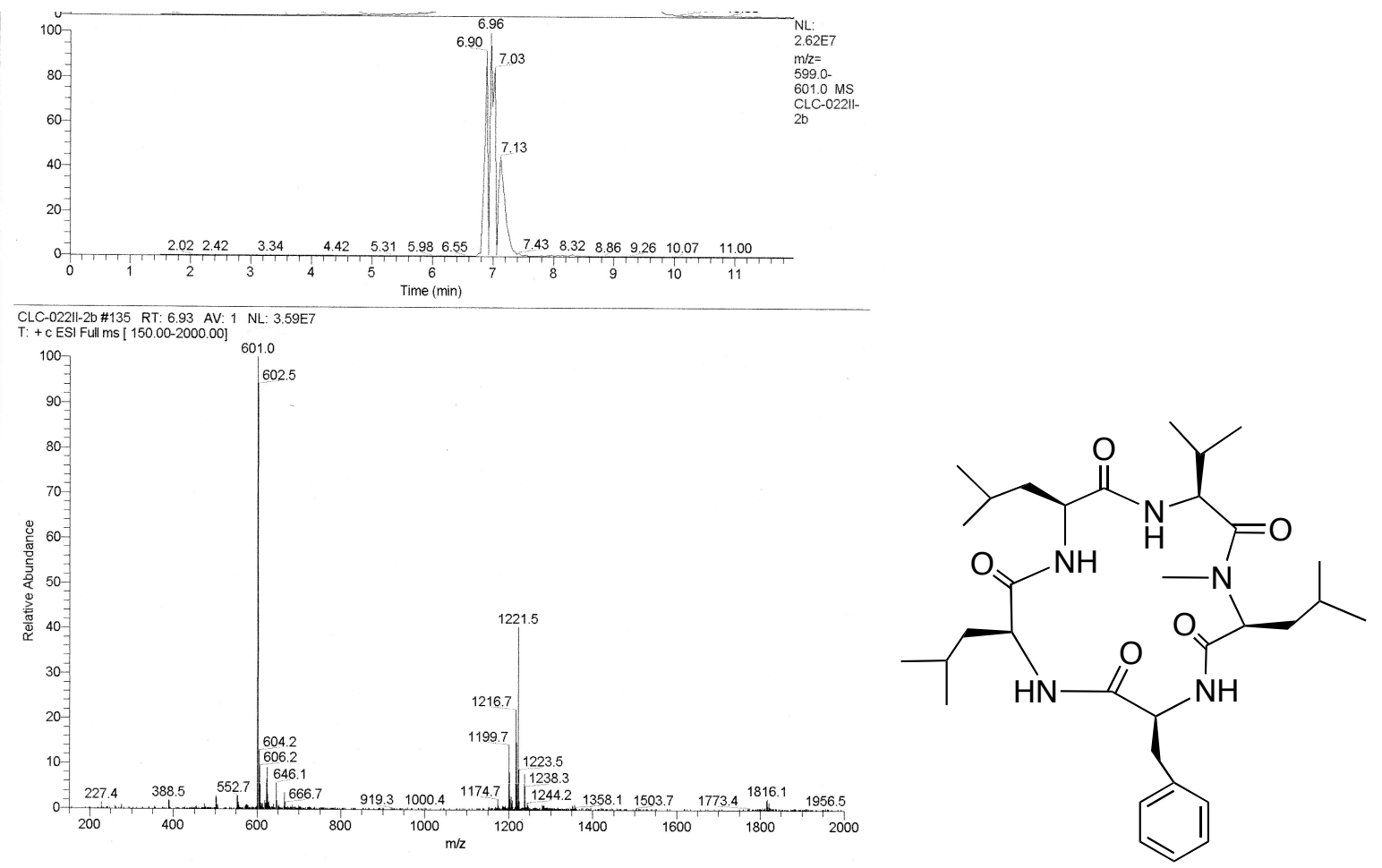

\section{Compound C Cyclized 1a-2c-3a-4a-5a (MW=599)}
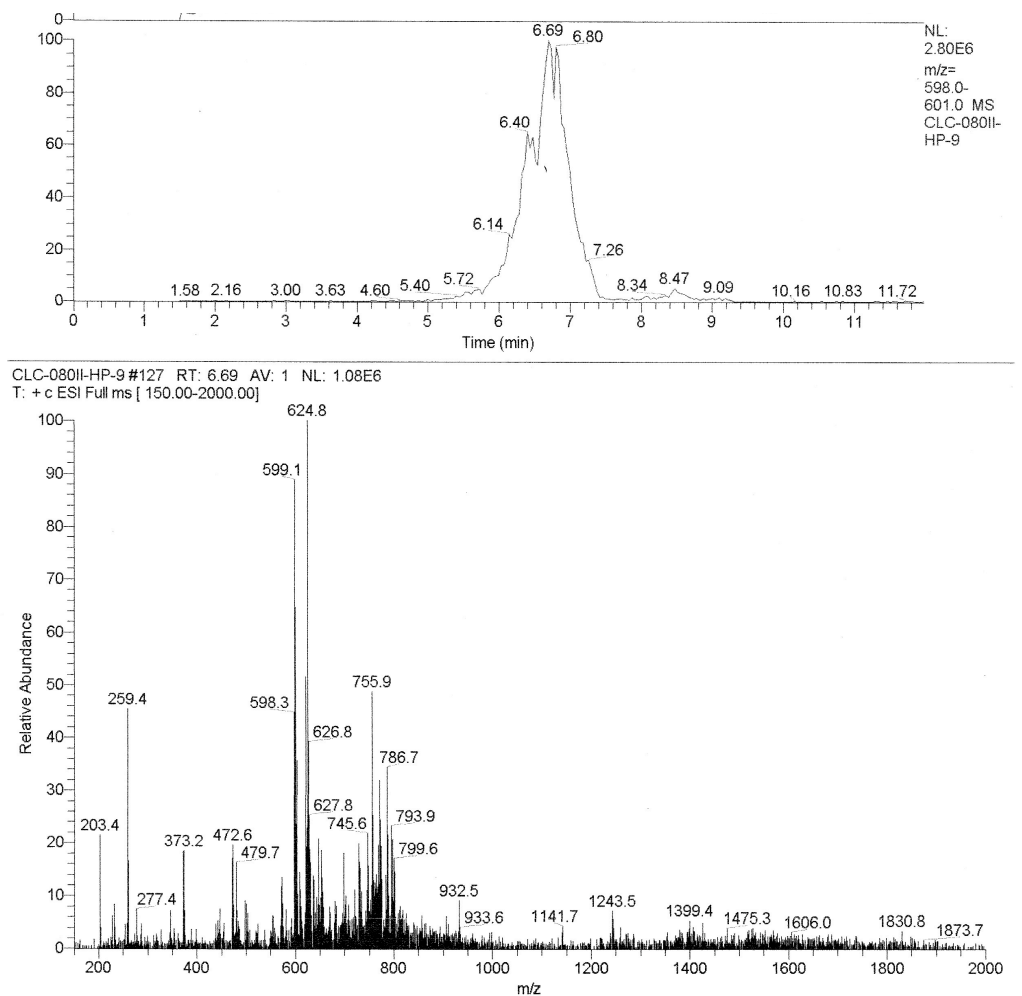

Compound D Cyclized 1b-2d-3b-4b-5b (MW=599)

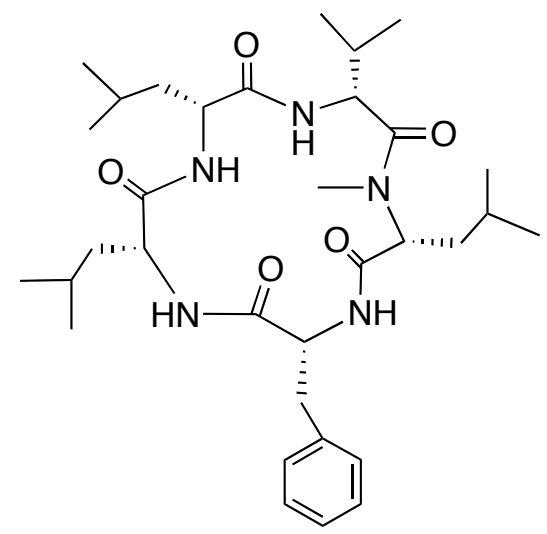



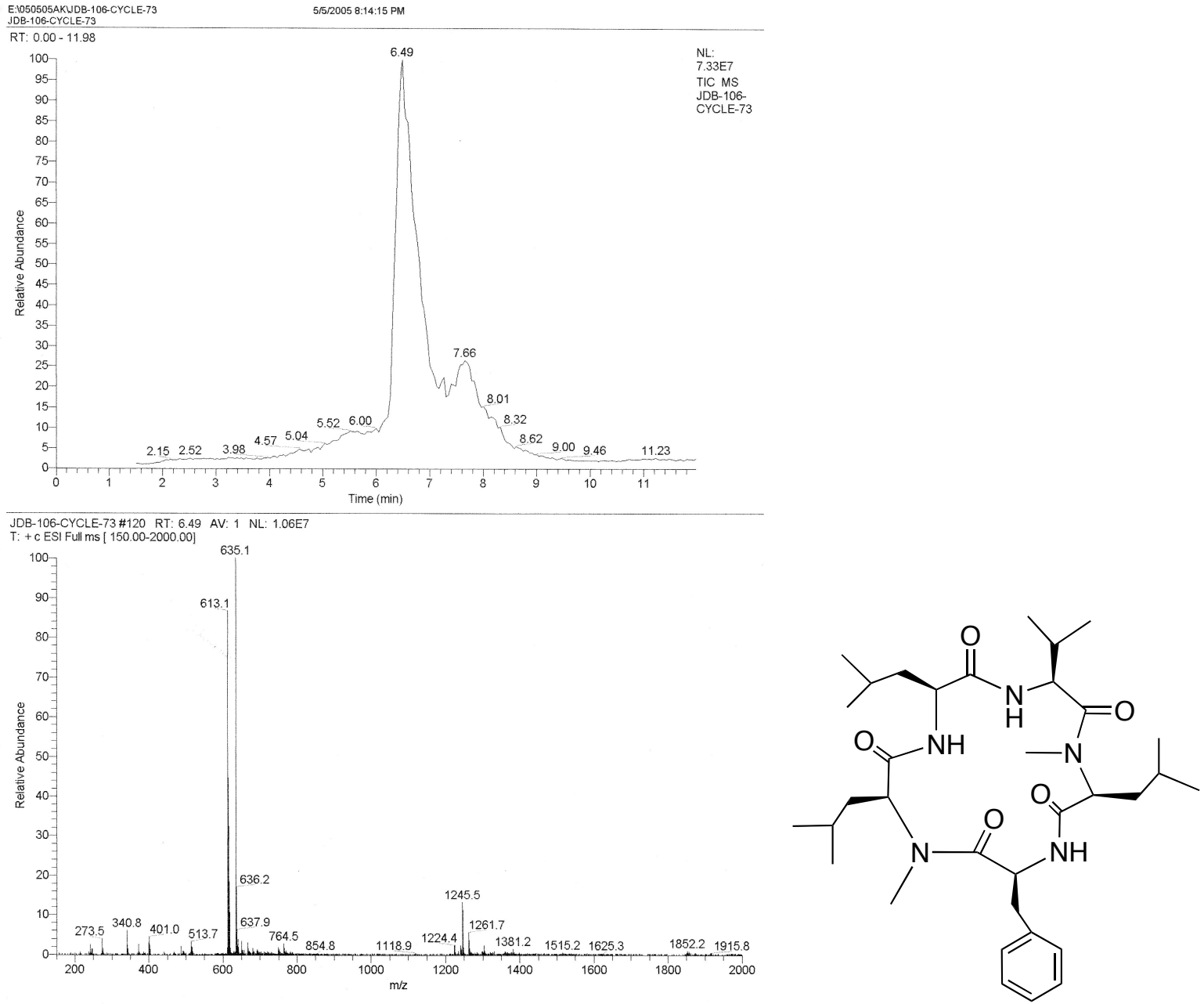

Compound E Cyclized 1a-2c-3a-4a-5c (MW=613) 

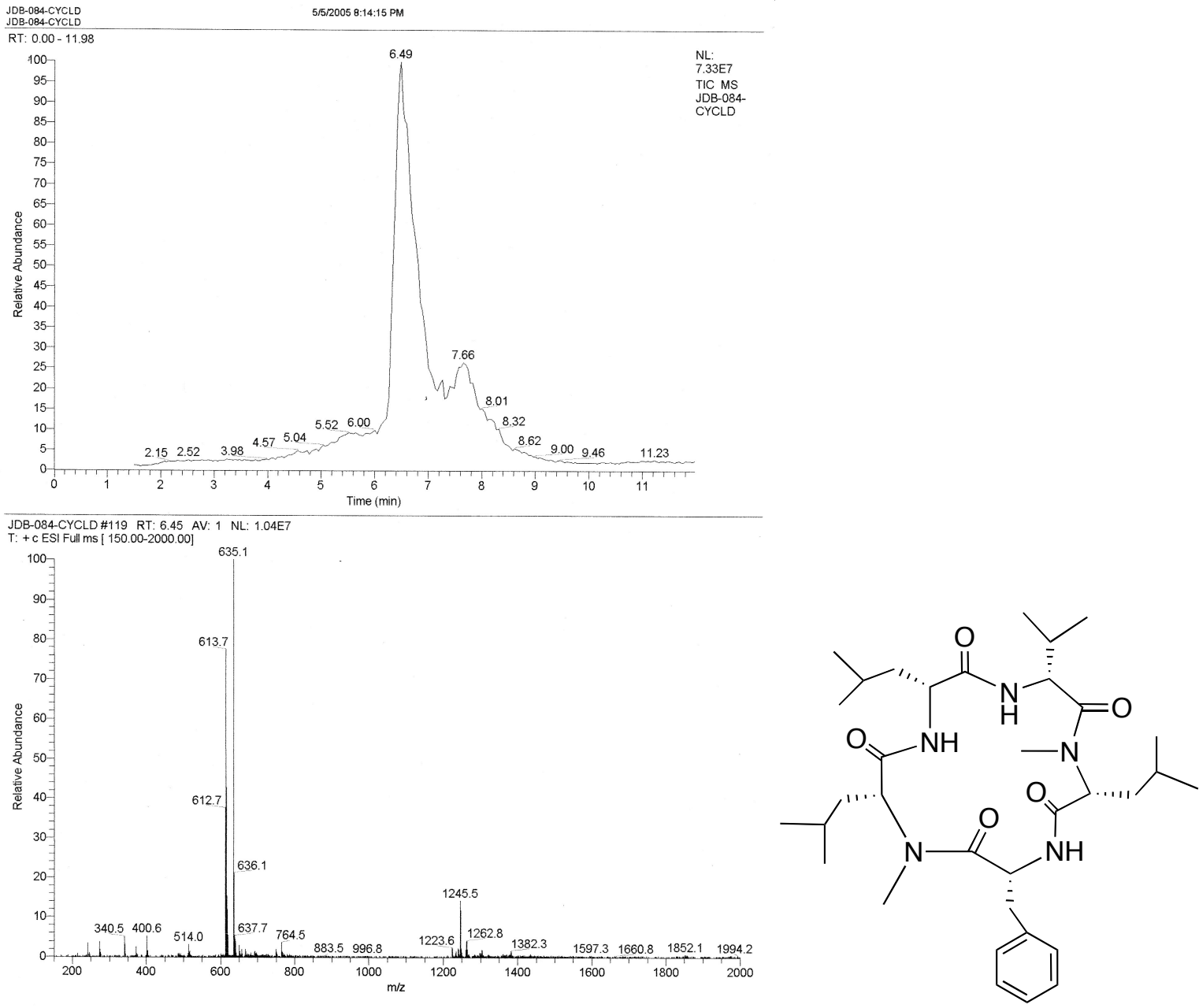

\section{Compound F Cyclized 1b-2d-3b-4b-5d (MW=613)}



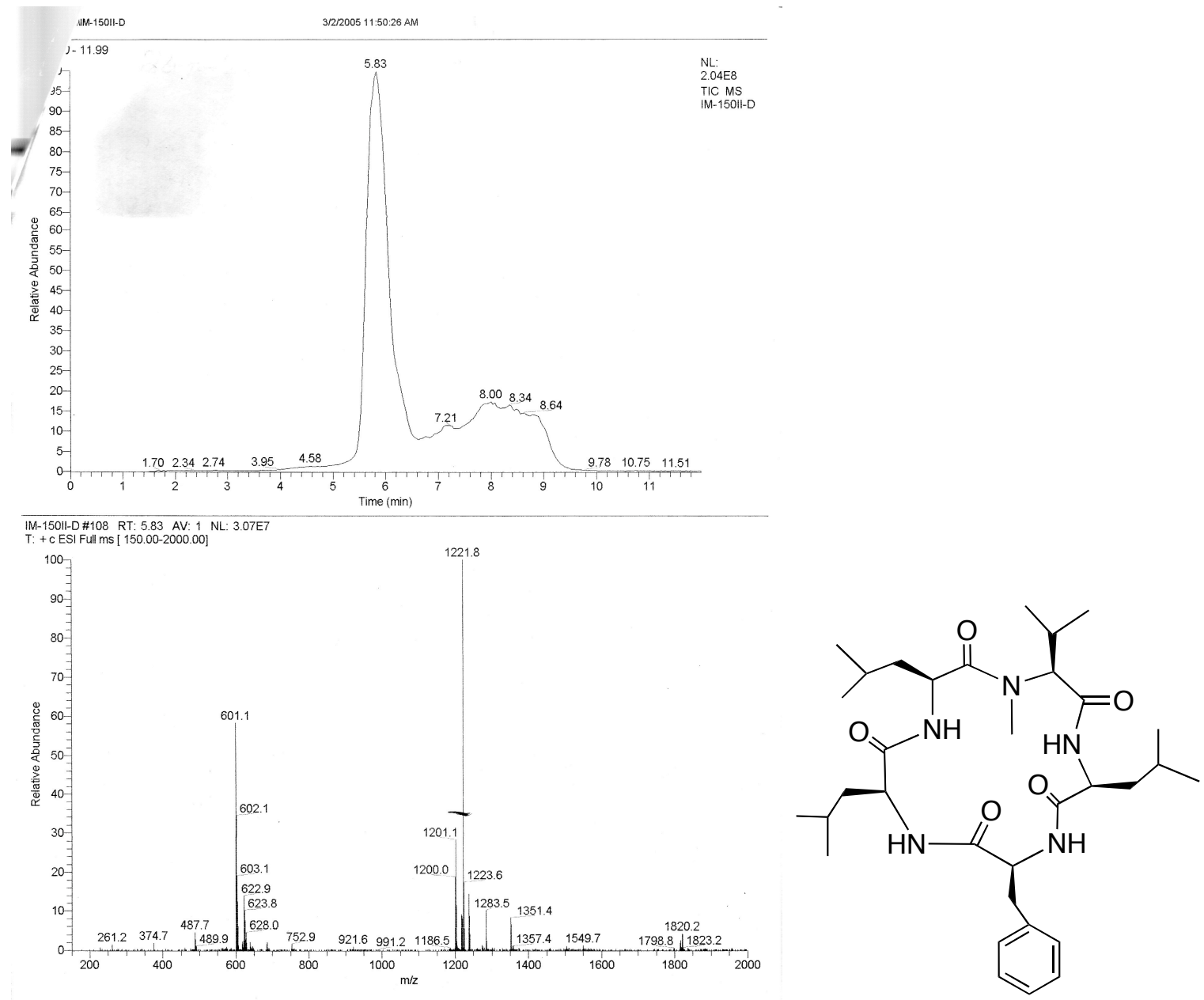

\section{Compound G Cyclized 1a-2a-3c-4a-5a (MW=599)}



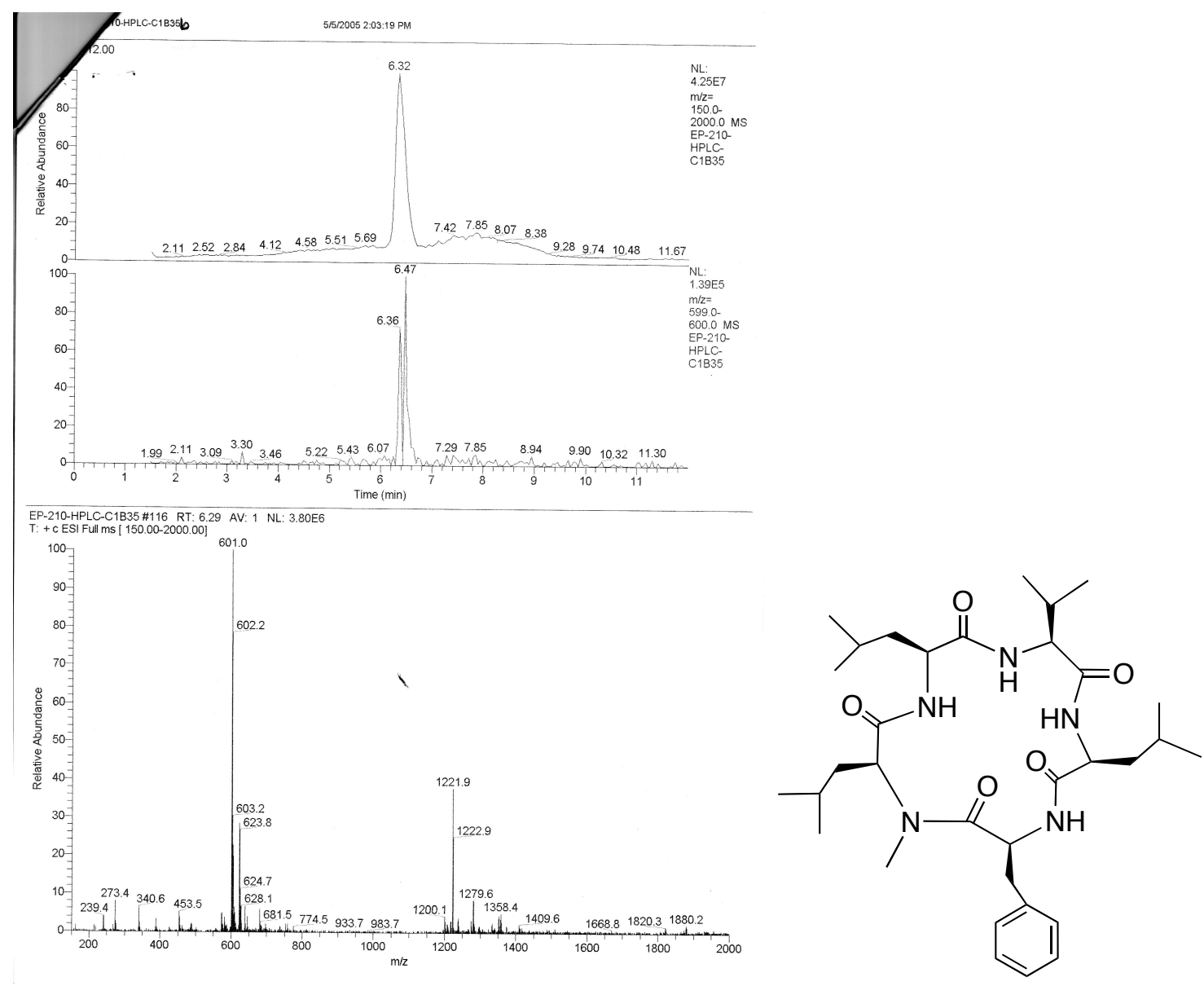

Compound H Cyclized 1a-2a-3a-4a-5c (MW=599) 

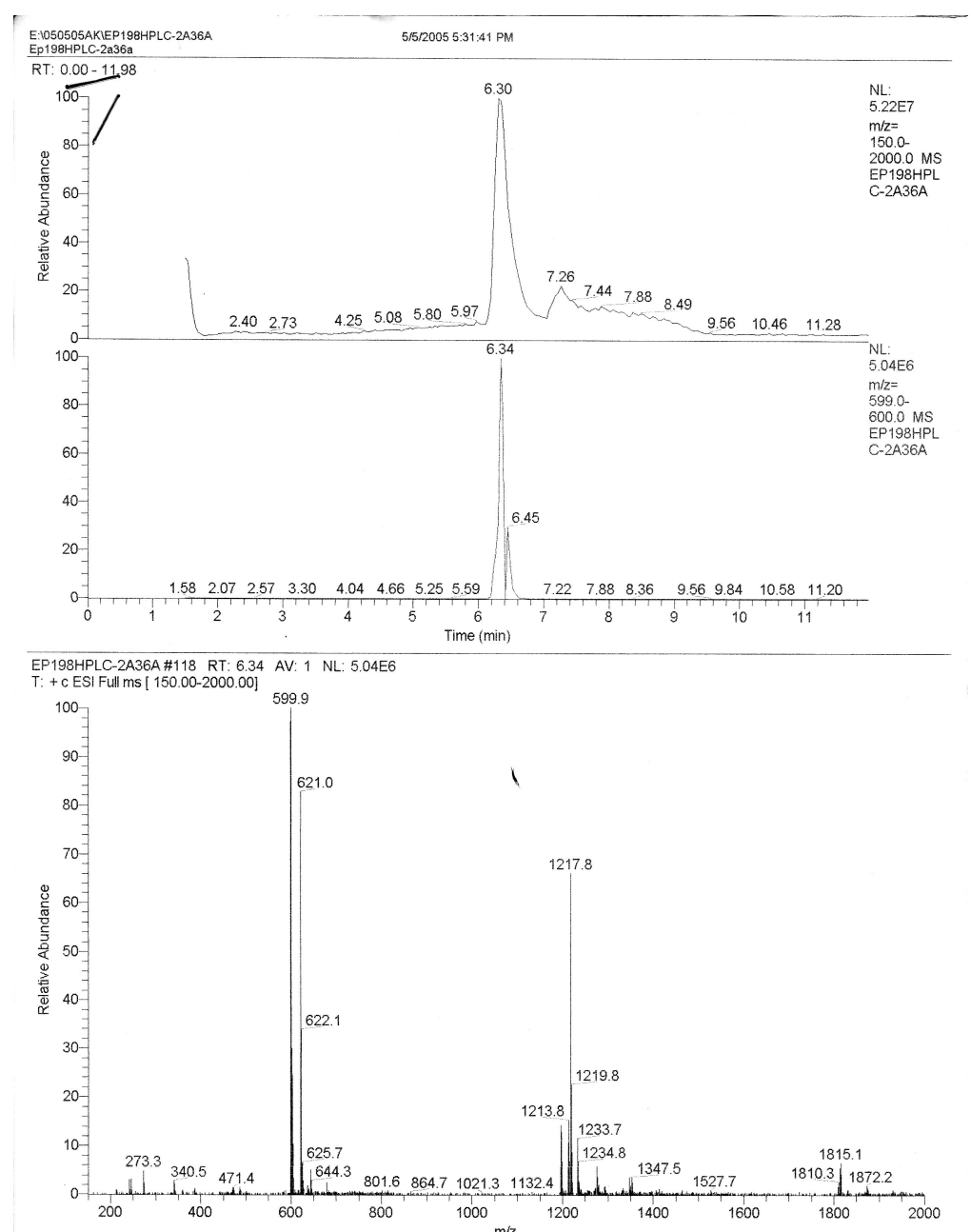

\section{Compound I Cyclized 1b-2b-3b-4b-5d (MW=599)}

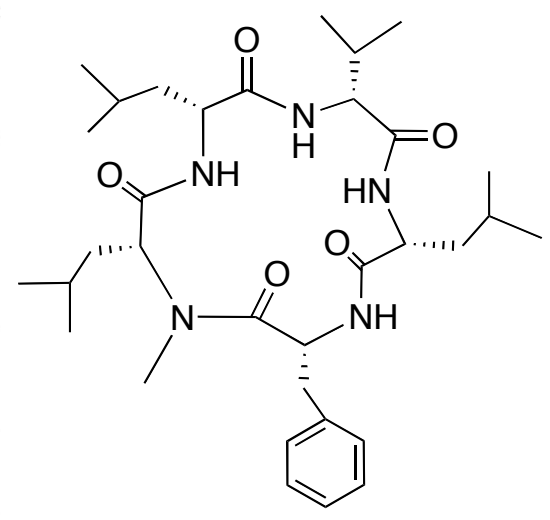



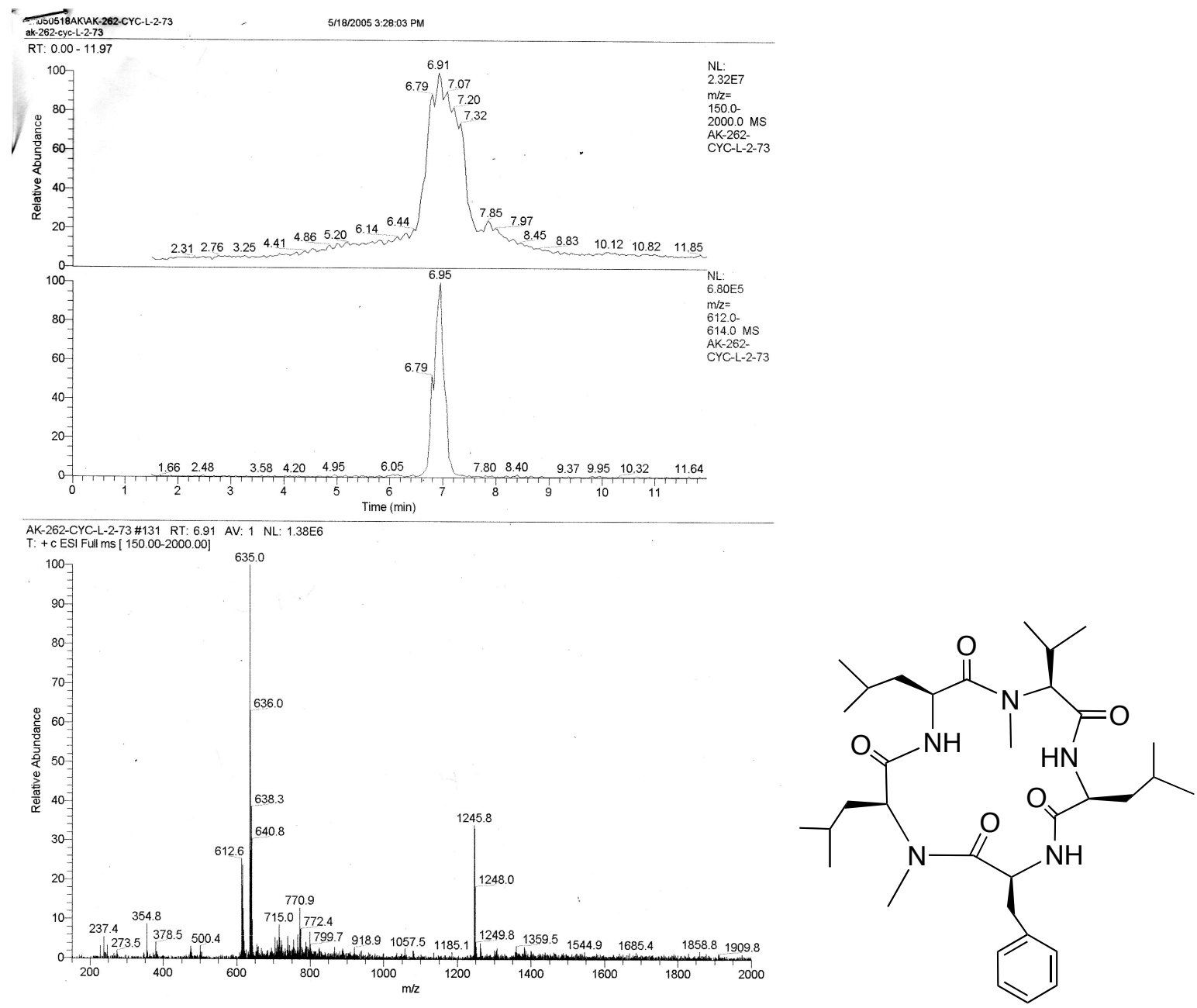

Compound J Cyclized 1a-2a-3c-4a-5c $(M W=612)$ 

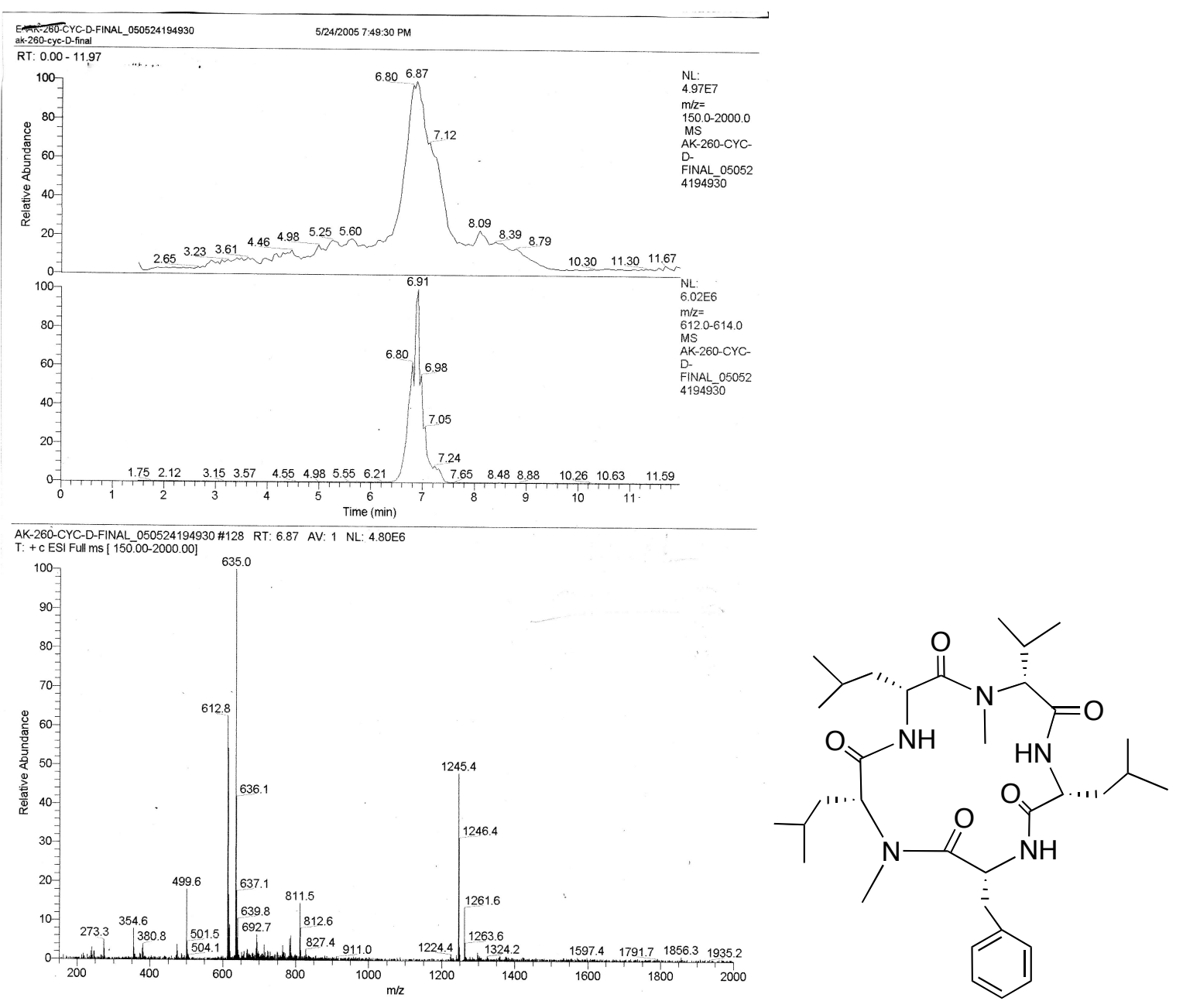

Compound K Cyclized 1b-2b-3d-4b-5d (MW=612) 


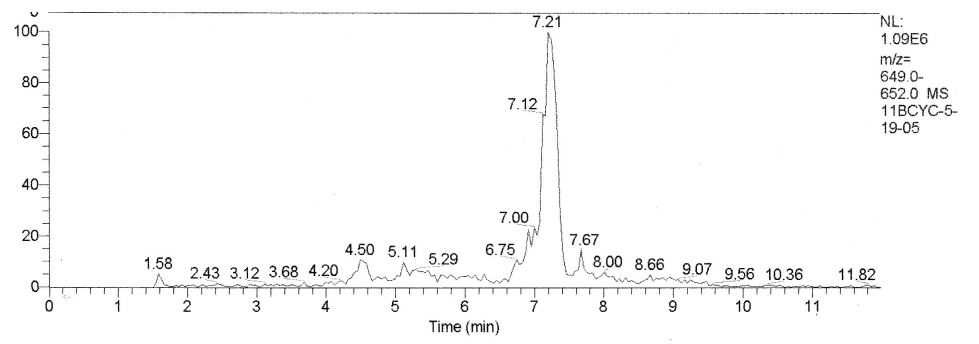

11BCYC-5-19-05\#132 RT: 7.17 AV: 1 NL: 3.49E5
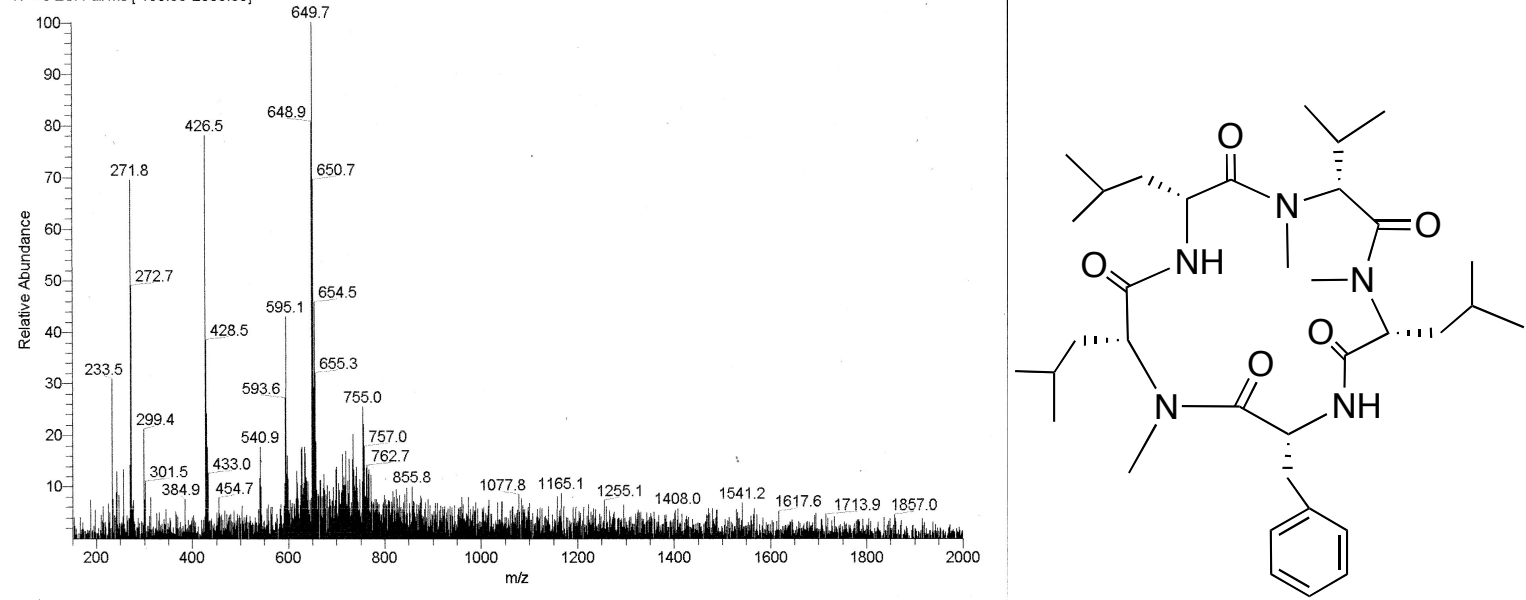

Compound L Cyclized 1b-2d-3d-4b-5d (MW=626) 

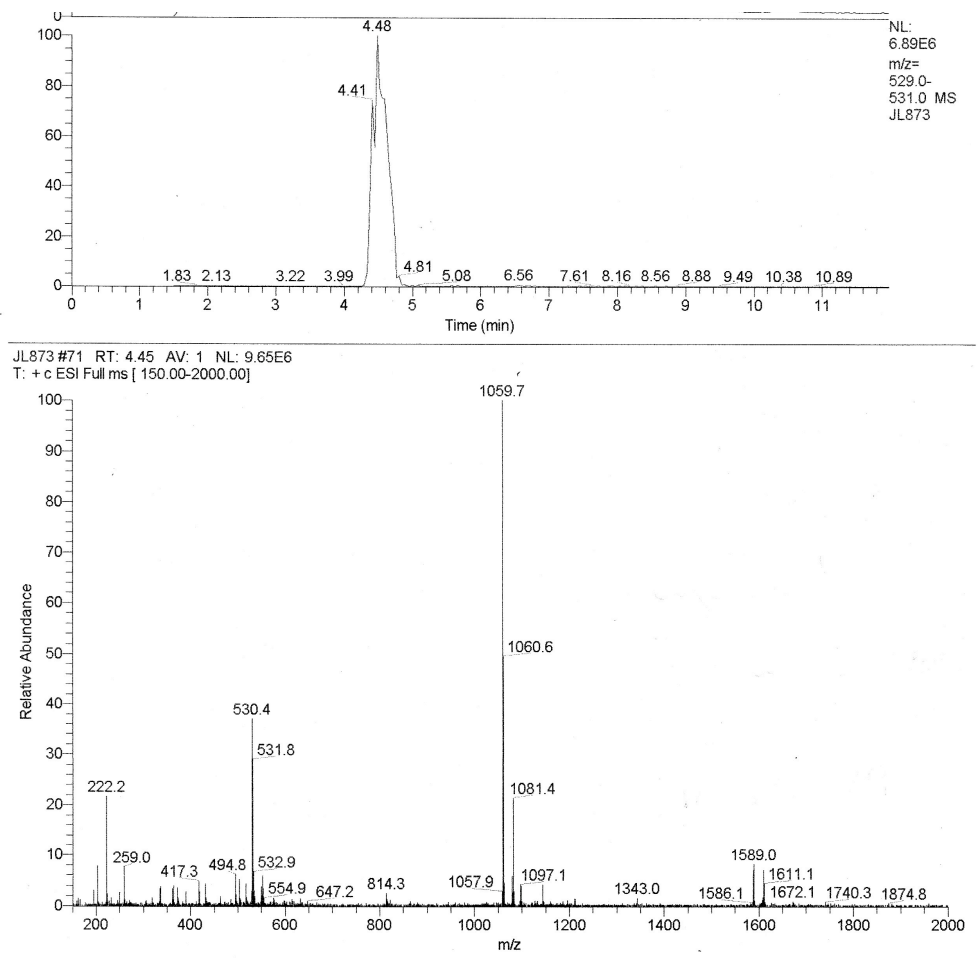

Compound M Cyclized 1a-2e-3a-4a-5a $\quad(M W=529)$

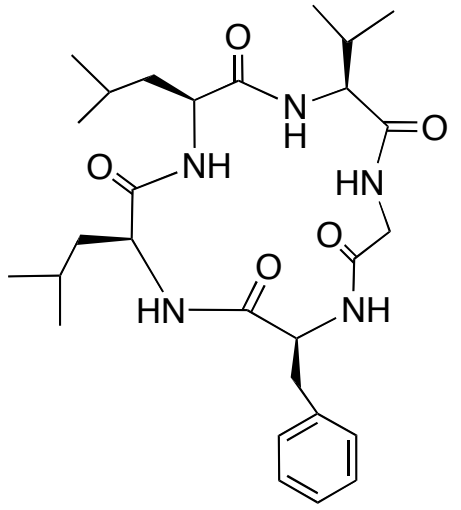



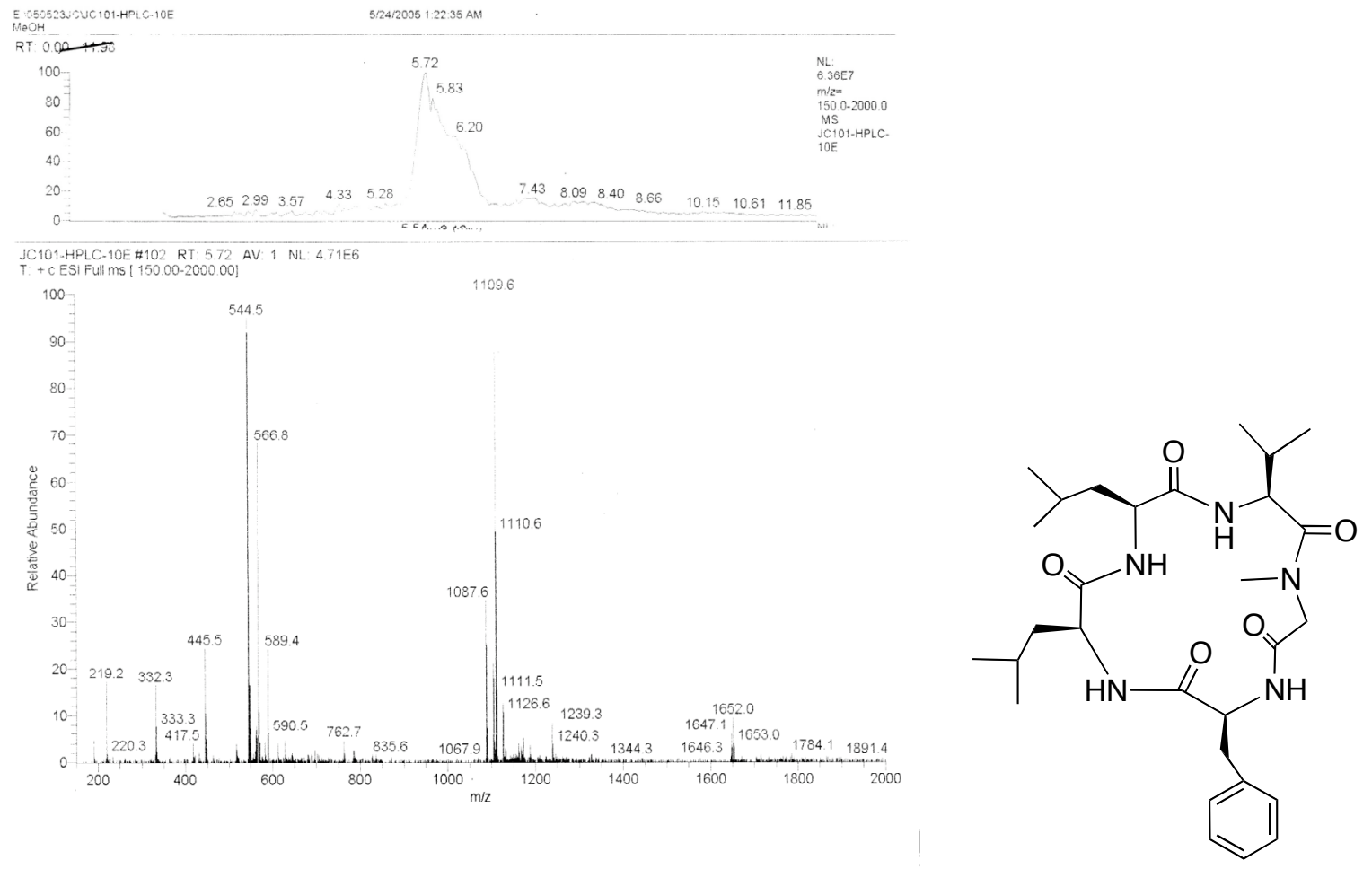

Compound N Cyclized 1a-2f-3a-4a-5a $(M W=543)$ 